\title{
Empty singularities in higher-dimensional Gravity
}

\author{
Ricardo E. Gamboa Saraví
}

Received: date / Revised version: date

\begin{abstract}
We study the exact solution of Einstein's field equations consisting of a $(n+2)$-dimensional static and hyperplane symmetric thick slice of matter, with constant and positive energy density $\rho$ and thickness $d$, surrounded by two different vacua. We explicitly write down the pressure and the external gravitational fields in terms of $\rho$ and $d$, the pressure is positive and bounded, presenting a maximum at an asymmetrical position. And if $\sqrt{\rho} d$ is small enough, the dominant energy condition is satisfied all over the spacetime. We find that this solution presents many interesting features. In particular, it has an empty singular boundary in one of the vacua.
\end{abstract}

PACS 04.20.JB

\section{Introduction}

We have recently pointed out that solutions of Einstein's field equations presenting an empty (free of matter) repelling singular boundary where spacetime curvature diverges can occur in four dimensions [1,2,3,4. These singularities are not the sources of the fields, but rather, they arise owing to the attraction of distant matter.

The solution described in [1] is the gravitational field of a static and plane symmetric distribution of matter lying below $z=0$. Because of the symmetry required and depending on the properties of the matter, the exterior solution can only turn out to be either Rindler's flat spacetime or Taub's plane vacuum one [5]. Assuming the latter, which has a singular boundary at a finite height, it is showed that only vertical null geodesics just touch the singularity and

E-mail: quique@fisica.unlp.edu.ar

Departamento de Física, Facultad de Ciencias Exactas, Universidad Nacional de La Plata and IFLP, CONICET. C.C. 67, 1900 La Plata, Argentina. 
bounce, whereas non-vertical null ones as well as massive particles bounce before getting to it. Furthermore, later on, we rigorously show that the Cauchy problem for wave propagation in this vacuum is well-posed, if we only demand the waves to have finite energy, although no boundary condition is required 6 . And those waves completely reflect at the singularity. Due to these properties we also call this kind of singularities white walls.

A detailed study of the inner solution for a static and plane symmetric relativistic perfect fluid obeying an equation of state such that $\rho$ and $p$ are proportional to each other is presented in [2]. In this case, it is not possible to match this solution to a vacuum one since the pressure does not vanish at any regular point. Nevertheless, it already exhibits by itself such a property because it is a semi-infinite spacetime where pressure and density vanish at a singular boundary as well as at infinite.

An exhaustive analysis of the properties of the internal solution for a static plane symmetric relativistic perfect incompressible fluid with positive density, originally found by Taub in [7, is shown in [3. We found that this solution finishes up down below at an inner singularity at finite depth $d$. This solution essentially depends on only one parameter $\kappa$, and depending on its value, it turns out to be gravitationally attractive, neutral or repulsive.

We analyze the $C^{1}$-matching of this inner solution and vacua in [4]. In particular, we consider a non-singular slice of the inner solution with thickness $d$ $\left(0<d<\sqrt{\frac{\pi}{24 \rho}}\right)$ surrounded by two external vacua. The solution turns out to be attractive and remarkably asymmetric: the "upper" solution is Rindler's vacuum, whereas the "lower" one is the singular part of Taub's plane symmetric solution which finishes up down below at a boundary where spacetime curvature diverges. We explicitly write down the pressure and the external gravitational fields in terms of $\rho$ and $d$, the pressure is positive and bounded presenting a maximum at an asymmetrical position. And if $0<\sqrt{6 \pi \rho} d<1.527 \ldots$, the dominant energy condition is satisfied all over the spacetime. This exact and complete (matter matched to vacua) solution clearly shows how the attraction of distant matter can shrink space-time in such a way that it ends up at an empty singular boundary.

Due to the plane symmetry these solutions may look somewhat unnatural, nevertheless they can give an idea of the qualitative features that could arise in General Relativity, and so, of possible properties of realistic solutions.

The possibility that spacetime may have more than four dimensions is now a standard assumption in High Energy Physics. Indeed, most theories attempting to unify all the fundamental interactions and quantize gravity require a higher dimensional spacetime, where higher-dimensional gravity (HDG) plays a decisive roll. Therefore, among the reasons it should be interesting to study HDG we may mention that

- String and M-theories contain gravity and requiere the existence of more than four space-time dimensions, namely ten or eleven. 
- The AdS/CFT correspondence [8] states that the physics of gravity in a higher-dimensional spacetime, is equivalent to a certain quantum field theory which is defined on the boundary (for a review see, for example, [9]).

- According to world-brane models the observable universe could be $1+$ 3-surface (the "brane") embeded in a high-dimensional sapacetime (the "bulk"), with Standard Model particles and fields trapped on the brane while gravity is free to acces the bulk (for a review see, for example, [10]).

- HDR is also interesting from a purely mathematical point of view. It is noteworthy that some known results of general relativity, turn out to be quite specific to four dimensions. For example, existence of stable bound orbits or black hole no-hair theorem [11.

It is well known that Einstein's Gravity gets weaker as the number of space-time dimensions increases. For instance, stable bound Keplerian orbits do not exist in space-times of more than four dimensions [12, or also, the degree of compactification of spherical stars diminishes as the dimensionality of space-time raises 13 .

On the other hand, HDG seems to be richer. Indeed, a much larger variety of asymptotically flat stationary black holes exists in spacetimes with extra dimensions. For instance, besides the Myer-Perry solution [14, in five dimensions there exist black rings, black saturns, ... (for a review see, for example, [15]).

Thus, it would be worthwhile finding out whether empty singular boundaries can also arise in higher-dimensional Gravity, and the aim of this paper is to explore this issue.

In this work, we show that such singularities may occur in space-times of $n+2$ dimensions $(n \geq 2)$ by constructing an explicit solution.

The solution to be described is a $(n+2)$-dimensional static and hyperplane symmetric space-time formed by a thick slice of matter, with constant and positive energy density $\rho$ and thickness $d$, surrounded by two vacua. It turns out that this solution shares all the main features of the one that was studied in [4] for the $n=2$ case. In particular, it has an empty singular boundary in one of the vacua.

For $n \geq 2$, we adopt the convention in which the metric of the $(n+2)$ dimensional space-time has signature $(-+\ldots++)$ and the system of units in which the speed of light $c=1$, and $G$ is the $(n+2)$-dimensional Newton's gravitational constant.

\section{The symmetry and Einstein's field equations}

We here consider solutions of Einstein's equations corresponding to a static and hyperplane symmetric distribution of matter. That is, they must be invariant under $n$ translations and under rotations in $n(n-1) / 2$ planes. The matter we consider is a perfect fluid with stress-energy tensor

$$
T_{a b}=(\rho+p) u_{a} u_{b}+p g_{a b},
$$


where $u^{a}$ is the velocity of fluid elements.

Due to the required symmetry, as shown in $\mathrm{A}$, we can find coordinates such that the line element can be written as

$$
d s^{2}=-\mathcal{G}(z)^{2} d t^{2}+e^{2 V(z)}\left(\left(d x^{1}\right)^{2}+\ldots+\left(d x^{n}\right)^{2}\right)+d z^{2} .
$$

The non identically vanishing components of the Einstein tensor are

$$
\begin{array}{r}
G_{t t}=-n \mathcal{G}^{2}\left(V^{\prime \prime}+\frac{n+1}{2} V^{\prime 2}\right)(2) \\
G_{11}=\ldots=G_{n n}=e^{2 V}\left(\frac{\mathcal{G}^{\prime \prime}}{\mathcal{G}}+(n-1) \frac{\mathcal{G}^{\prime}}{\mathcal{G}} V^{\prime}+(n-1) V^{\prime \prime}+\frac{n(n-1)}{2} V^{\prime 2}\right)(3) \\
G_{z z}=n V^{\prime}\left(\frac{\mathcal{G}^{\prime}}{\mathcal{G}}+\frac{n-1}{2} V^{\prime}\right)(, 4)
\end{array}
$$

where a prime $\left({ }^{\prime}\right)$ denotes differentiation with respect to $z$.

On the other hand, since the fluid must be static, $u_{a}=(-\mathcal{G}, 0, \ldots, 0)$, so

$$
T_{a b}=\operatorname{diag}\left(\rho \mathcal{G}^{2}, p e^{2 V}, \ldots, p e^{2 V}, p\right),
$$

where $\rho$ and $p$ can depend only on $z$. Thus, Einstein's equations, i.e., $G_{a b}=$ $4 \pi G \frac{n}{n-1} T_{a b}$ 1 1 , are

$$
\begin{aligned}
V^{\prime \prime}+\frac{n+1}{2} V^{\prime 2} & =-\frac{4 \pi G}{n-1} \rho, \\
\frac{\mathcal{G}^{\prime \prime}}{\mathcal{G}}+(n-1) \frac{\mathcal{G}^{\prime}}{\mathcal{G}} V^{\prime}+(n-1) V^{\prime \prime}+\frac{n(n-1)}{2} V^{\prime 2} & =\frac{4 \pi n G}{n-1} p, \\
\frac{\mathcal{G}^{\prime}}{\mathcal{G}} V^{\prime}+\frac{(n-1)}{2} V^{\prime 2} & =\frac{4 \pi G}{n-1} p .
\end{aligned}
$$

Moreover, $\nabla_{a} T^{a b}=0$ yields

$$
p^{\prime}=-(\rho+p) \frac{\mathcal{G}^{\prime}}{\mathcal{G}} .
$$

Of course, due to Bianchi's identities, equations (6)-(9) are not independent.

Regarding the mirror symmetry, it can be shown that, independently of the equation of state and the dimension of space-time, the solution cannot have a "plane" of symmetry in a region where $\rho(z) \neq 0$ and $p(z) \geq 0$. In order to see this, let us assume that $z=z_{s}$ is that "plane", then it must hold that $\mathcal{G}^{\prime}=V^{\prime}=p^{\prime}=\rho^{\prime}=0$ at $z_{s}$, and so from (8) we get that also $p\left(z_{s}\right)=0$. Now, by differentiating (9) and using (6) and (7), we obtain $p^{\prime \prime}\left(z_{s}\right)=-4 \pi G \rho^{2}<0$.

1 We have set the normalization constant such that, in the Newtonian limit, they lead to $\nabla^{2} \Phi=-\frac{1}{2} \nabla^{2}\left(1+g_{t t}\right)=4 \pi G \rho$. 


\section{Solution with constant and positive density $\rho$}

In this section, we consider the solution for matter having constant energy density $\rho>0$. Proceeding analogously as in 3] and [4, where the four-dimensional case is considered, we find the solution

$$
\begin{gathered}
p(z)=\frac{C_{p}}{\mathcal{G}(z)}-\rho, \\
V(z)=\ln \left(C_{1} \sin u\right)^{\frac{2}{n+1}},
\end{gathered}
$$

and

$$
\mathcal{G}=C_{3} \frac{\cos u}{(\sin u)^{\frac{n-1}{n+1}}}+\frac{C_{p}}{\rho} \frac{(n+1)}{(3 n+1)} \sin ^{2} u{ }_{2} F_{1}\left(1, \frac{n}{n+1} ; \frac{5 n+3}{2(n+1)} ; \sin ^{2} u\right),
$$

where $u=\sqrt{2 \pi G \frac{n+1}{n-1} \rho} z+C_{2},{ }_{2} F_{1}(a, b ; c ; z)$ is the Gauss hypergeometric function (see for example [16]) and $C_{p}, C_{1}, C_{2}$ and $C_{3}$ are arbitrary constants.

Therefore, the line element (1) becomes

$$
d s^{2}=-\mathcal{G}(z)^{2} d t^{2}+\left(C_{1} \sin u\right)^{\frac{4}{n+1}}\left(\left(d x^{1}\right)^{2}+\ldots+\left(d x^{n}\right)^{2}\right)+d z^{2} .
$$

This solution is the higher dimensional generalization of the one found by Taub 7 .

By contracting the Ricci tensor, we get

$$
R(z)=\frac{8 \pi G}{n-1}(\rho-(n+1) p(z))
$$

On the other hand, we see that the metric has a space-time curvature singularity when $\sin u=0$, since straightforward computation of the scalar quadratic in the Riemann tensor yields

$$
\begin{gathered}
R_{a b c d} R^{a b c d}=4\left(\frac{\mathcal{G}^{\prime \prime 2}}{\mathcal{G}^{2}}+n \frac{\mathcal{G}^{\prime 2}}{\mathcal{G}^{2}} V^{\prime 2}\right)+4 n\left(V^{\prime \prime 2}+2 V^{\prime \prime} V^{\prime 2}+\frac{n+1}{2} V^{\prime 4}\right) \\
=\quad 64 \pi^{2} G^{2} \rho^{2} \frac{n^{2}}{n^{2}-1}\left(\frac{1}{\sin ^{4} u}+\frac{(n+1)^{2}}{n^{2}(n-1)}\left(1+\frac{p}{\rho}\right)^{2}\right. \\
\left.-\frac{4(n+1)}{n^{2}(n-1)}\left(1+\frac{p}{\rho}\right)+\frac{2(n+2)}{n^{2}(n-1)}\right),
\end{gathered}
$$

so $R_{a b c d} R^{a b c d} \rightarrow \infty$ when $\sin u \rightarrow 0$. 


\section{Vacuum limits}

From the solution found above, we can obtain vacuum ones as a limit. In fact, when $C_{p}=0$, it is clear from (10) that $p(z)=-\rho$, and the solution (13) turns out to be a vacuum solution with a cosmological constant $\Lambda=\frac{4 \pi n G}{n-1} \rho$

$$
\begin{array}{r}
d s^{2}=-\cos ^{2} u(\sin u)^{-2\left(\frac{n-1}{n+1}\right)} d t^{2}+(\sin u)^{\frac{4}{n+1}}\left(\left(d x^{1}\right)^{2}+\ldots+\left(d x^{n}\right)^{2}\right)+d z^{2} \\
-\infty<t<\infty, \quad-\infty<x^{1}<\infty, \quad \ldots, \quad-\infty<x^{n}<\infty, \quad 0<u<\pi,
\end{array}
$$

where $u=\sqrt{\frac{(n+1) \Lambda}{2 n}} z+C_{2}$. We get from (14) that it is a space-time with constant scalar curvature $\frac{2(n+2)}{n} \Lambda$, and from (15) we get that

$$
R_{a b c d} R^{a b c d}=4\left(\frac{n-1}{n+1}\right) \Lambda^{2}\left(\frac{1}{\sin ^{4} u}+\frac{2(n+2)}{(n-1) n^{2}}\right) .
$$

This solution is the generalization of the one what was found for $n=2$ in [17.

Now, we take the limit $\Lambda \rightarrow 0(\rho \rightarrow 0)$. By setting $C_{2}=\pi-\frac{n-1}{g_{T}} \sqrt{\frac{\Lambda}{2 n(n+1)}}$ and an appropriate rescaling of the coordinates $\left\{t, x^{1}, \ldots, x^{n}\right\}$, we can readily see that when $\Lambda \rightarrow 0$, (16) becomes

$$
\begin{gathered}
d s^{2}=-\left(1-\frac{n+1}{n-1} g_{T} z\right)^{-2\left(\frac{n-1}{n+1}\right)} d t^{2} \\
+\left(1-\frac{n+1}{n-1} g_{T} z\right)^{\frac{4}{n+1}}\left(\left(d x^{1}\right)^{2}+\ldots+\left(d x^{n}\right)^{2}\right)+d z^{2}, \\
-\infty<t<\infty,-\infty<x^{1}<\infty, \ldots,-\infty<x^{n}<\infty, 0<1-\frac{n+1}{n-1} g_{T} z<\infty,
\end{gathered}
$$

where $g_{T}$ is an arbitrary constant. In (18), the coordinates have been chosen in such a way that the solution describes a homogeneous gravitational field $g_{T}$ pointing in the negative $z$-direction in a neighborhood of $z=0$. The metric (18) is the generalization Taubs's vacuum plane solution [18]. Notice that, in this case, (17) becomes

$$
R_{a b c d} R^{a b c d}=\frac{16 n^{2}\left(n^{2}-1\right) g_{T}^{4}}{(n-1)^{4}} \frac{1}{\left(1-\frac{n+1}{n-1} g_{T} z\right)^{4}},
$$

hence, this space-time finishes at a singular boundary at $z=\frac{n-1}{(n+1) g_{T}}$.

On the other hand, by setting $C_{2}=\frac{1}{g_{R}} \sqrt{\frac{(n+1) \Lambda}{2 n}}+\frac{\pi}{2}$ and an appropriate rescaling of the coordinate $t$, we can readily see that when $\Lambda \rightarrow 0$, (16) becomes

$$
\begin{gathered}
d s^{2}=-\left(1+g_{R} z\right)^{2} d t^{2}+\left(d x^{1}\right)^{2}+\ldots+\left(d x^{n}\right)^{2}+d z^{2}, \\
-\infty<t<\infty, \quad-\infty<x^{1}<\infty, \quad \ldots, \quad-\infty<x^{n}<\infty, \quad-\frac{1}{g_{R}}<z<\infty,
\end{gathered}
$$


where $g_{R}$ is an arbitrary constant, and the coordinates have been chosen in such a way that the solution also describes a homogeneous gravitational field $g_{R}$ pointing in the negative $z$-direction in a neighborhood of $z=0$. The metric (20) is a $(n+2)$-dimensional Rindler's flat space-time.

Therefore, there are two very different vacua admitting the imposed symmetry, and both of them will be required to match to the inner solution.

\section{Properties of the interior solution}

Here we confine our attention to positive values of $\rho$ and $C_{p} \neq 0$. Without loss of generality, proceeding as in [3] and [4 by an appropriate rescaling of the coordinates $\left\{t, x^{1}, \ldots, x^{n}\right\}$, we can transform away the irrelevant parameters and see that the solution essentially depends on two parameters $\rho$ and $\kappa$. The line element (13) reads

$$
\begin{array}{r}
d s^{2}=-\mathcal{G}(z)^{2} d t^{2}+(\sin u)^{\frac{4}{n+1}}\left(\left(d x^{1}\right)^{2}+\ldots+\left(d x^{n}\right)^{2}\right)+d z^{2} . \\
-\infty<t<\infty, \quad-\infty<x^{1}<\infty, \quad \ldots, \quad-\infty<x^{n}<\infty \\
0<u=\sqrt{2 \pi G \frac{n+1}{n-1} \rho} z+C_{2} \leq \pi / 2,
\end{array}
$$

where

$$
\mathcal{G}(z)=\frac{\left(\kappa-\kappa_{\text {crit }}\right) \cos u+{ }_{2} F_{1}\left(-\frac{1}{2}, \frac{1-n}{2(n+1)} ; \frac{1}{2} ; \cos ^{2} u\right)}{(\sin u)^{\frac{n-1}{n+1}}}
$$

and

$$
\kappa_{\text {crit }}=\frac{\sqrt{\pi} \Gamma\left(\frac{3 n+1}{2(n+1)}\right)}{\Gamma\left(\frac{n}{n+1}\right)} .
$$

The pressure (10) becomes

$$
p(z)=\rho\left(\frac{1}{\mathcal{G}(z)}-1\right) .
$$

For $n=2$, in [3] and [4] we present a detailed study of the properties of the function $\mathcal{G}(z)$. Since for arbitrary $n$, the analysis completely parallels that one and its qualitative features do not depend on $n$, we restrict ourselves to point out the relevant results. The interested reader can find them following the same steps with slight modifications.

The properties of $\mathcal{G}$ drastically depend on the value of $\kappa$ (see Figs. 1 and 2 of Ref. (4). From (22) we see that, independently of the value of $\kappa, \mathcal{G}=1$ at $u=\pi / 2$ and then $p$ vanishes there. Moreover, we readily get from (22) that

$$
\left.\mathcal{G}^{\prime}(z)\right|_{u=\pi / 2}=\sqrt{\alpha}\left(\kappa_{\text {crit }}-\kappa\right)
$$


where $\alpha=2 \pi G \frac{n+1}{n-1} \rho$, and so the parameter $\kappa$ essentially governs the derivative of $\mathcal{G}$ at $u=\pi / 2$.

On the other hand, at $u=0, \mathcal{G}$ diverges if $\kappa \neq 0$, whereas it vanishes at that point if $\kappa=0$. Therefore, at $u=0, p=-\rho$ unless $\kappa=0$ in which case $p$ diverges.

If $\kappa \geq \kappa_{\text {crit }}$, in the interval $0<u \leq \pi / 2, \mathcal{G}(z)$ decreases monotonically from $\infty$ to 1 . Consequently, $p(z)$ is negative when $0 \leq u<\pi / 2$ and it increases monotonically from $-\rho$ to 0 and it satisfies $|p| \leq \rho$ all over the space-time (see Fig. 1(a) and Fig. 1(b) of Ref. 4]).

For $\kappa_{\text {crit }}>\kappa>0$, there is one (and only one) value $u_{m}$ where $\mathcal{G}(z)$ attains a local minimum. Hence, there is one (and only one) value $u_{0}\left(0<u_{0}<\pi / 2\right)$ such that $\left.\mathcal{G}(z)\right|_{u=u_{0}}=\left.\mathcal{G}(z)\right|_{u=\pi / 2}=1$, and then $\mathcal{G}(z)<1$ when $u_{0}<u<\pi / 2$. Since $\mathcal{G}(z)>0$, it is clear from (24) that $p(z)>0$ if $\mathcal{G}(z)<1$, and $p(z)$ reaches a maximum when $\mathcal{G}(z)$ attains a minimum. Hence, $p(z)$ grows from $-\rho$ to a maximum positive value when $u=u_{m}$ where it starts to decrease and vanishes at $u=\pi / 2$. Thus, $p(z)$ is negative when $0<u<u_{0}$ and positive when $u_{0}<u<\pi / 2$ (see Fig. 11).

It can be readily seen that, as $\kappa$ decreases from $\kappa_{\text {crit }}$ to $0, u_{m}$ moves to the left and the maximum value of $p(z) / \rho$ monotonically increases from 0 to $\infty$. It can be shown that for

$\kappa=\kappa_{\text {dec }}:=\kappa_{\text {crit }}-\sqrt{\frac{2 n}{n+1}}\left({ }_{2} F_{1}\left(-\frac{1}{2}, \frac{1-n}{2(n+1)} ; \frac{1}{2} ; \frac{n+1}{2 n}\right)-\frac{1}{2}\left(\frac{n-1}{2 n}\right)^{\frac{1-n}{2(n+1)}}\right)$

this maximum value gets 1 , and then $|p| \leq \rho$ all over the space-time for $\kappa \geq$ $\kappa_{d e c}$. Whereas, for $\kappa_{d e c}>\kappa>0$, although the pressure is bounded everywhere, there is a region of space-time where the dominant energy condition is violated.

For $\kappa \leq 0$, the pressure is unbounded (see Fig. 2 of Ref. [4]). If $\kappa<0$, we see from (15) that a new curvature singularity appears where $p$ diverges.

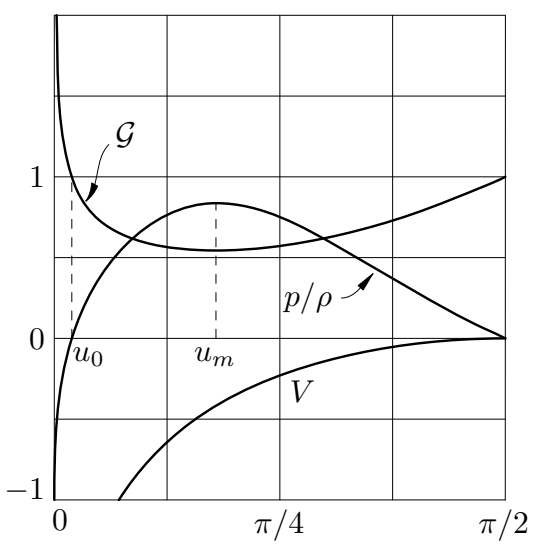

Fig. $1 \mathcal{G}(z), V(z)$ and $p(z) / \rho$ as functions of $u$, for $\kappa_{\text {crit }}>\kappa>\kappa_{\text {dec }}$. 


\section{$6 C^{1}$ matching of solutions}

In this section, we ensamble complete solutions by matching the interior solution and the vacuum ones. In order to avoid the appearance of undesirable superficial distribution of energy at the joint, we can readily see from (6) and (8) that the components of the metric tensor and their first derivatives must be continuous at the matching (hyper)surface.

Since for any value of $\kappa$, the pressure vanishes at $u=\pi / 2$, we can match the inner solution to a vacuum one there.

Now we see from (11) that the interior $V^{\prime}$ vanishes at $u=\pi / 2$. On the other hand, we can readily see that $V^{\prime}$ does not vanish at any finite point for Taub's vacuum (18), whereas $V^{\prime} \equiv 0$ for Ridler's vacuum (20). Therefore the inner solution can only be $C^{1}$-matched to the latter at $u=\pi / 2$.

Since the field equations are invariant under $z$-translation, we can choose to match the solutions at $z=0$ without losing generality, so we select $C_{2}=\pi / 2$. Thus, the inner singularity is located at $z=-\frac{\pi}{2 \sqrt{\alpha}}=-\sqrt{\frac{(n-1) \pi}{8(n+1) G \rho}}$.

Equations (20) and (21) show that at $z=0$ for both solutions, it holds that $g_{t t}=-1, g_{x^{i} x^{i}}=1$ and $\partial_{z} g_{x^{i} x^{i}}=0$. Moreover by using (25) we see that the continuity of $\partial_{z} g_{t t}$ at the boundary yields

$$
g_{R}=\sqrt{\alpha}\left(\kappa_{\text {crit }}-\kappa\right)
$$

which relates the external gravitational field $g_{R}$ with matter density $\rho$ and $\kappa$.

The last equation shows that if $\kappa>\kappa_{\text {crit }}, g$ is negative and the (hyper)slab turns out to be repulsive. If $\kappa=\kappa_{\text {crit }}$, it is gravitationally neutral, and the exterior is one half of Minkowski's space-time. If $\kappa<\kappa_{\text {crit }}$, it is attractive.

If $\kappa>0$, the depth of the slab is $\sqrt{\frac{(n-1) \pi}{8(n+1) G \rho}}$ independently of the value of $\kappa$. In this case, the pressure is finite anywhere, but it is negative deep below and $p=-\rho$ at the inner singularity (see Fig. 1 of Ref. 4]). But, as discussed above, only when $\kappa \geq \kappa_{d e c}$ is the condition $|p| \leq \rho$ everywhere satisfied.

If $\kappa \leq 0$, the pressure inside the slab is always positive, and it diverges deep below at the inner singularity (see Fig. 2 of Ref. [4]).

We have already seen that, in the case $\kappa_{\text {crit }}>\kappa>0$, the pressure also vanishes inside the slab at the point where $u=u_{0}$ (see Fig. 1). Therefore, in this case, by matching the slice of the interior solution $u_{0} \leq u \leq \pi / 2$ to a vacuum at $u_{0}$, we get an attractive slab surrounded by two vacua.

Clearly, the thickness of the slab is given by $d=\frac{\left(\pi / 2-u_{0}\right)}{\sqrt{\alpha}}$, and therefore $\left(0<d<\sqrt{\frac{(n-1) \pi}{8(n+1) G \rho}}\right)$. Now, since $\mathcal{G}(-d)=1$, from (22) we can write down $\kappa$ in terms of $d$ and $\rho$

$$
\kappa=\kappa_{\text {crit }}+\frac{(\cos (\sqrt{\alpha} d))^{\frac{n-1}{n+1}}-{ }_{2} F_{1}\left(-\frac{1}{2}, \frac{1-n}{2(n+1)} ; \frac{1}{2} ; \sin ^{2}(\sqrt{\alpha} d)\right)}{\sin (\sqrt{\alpha} d)}
$$

From the last expression we can easily see that $\kappa$ is a monotonically decreasing function of $d$. Therefore, taking into account (26), we find that, for every $n$, 
there exists a constant $C_{n}$ such that for $0<\sqrt{\rho} d<C_{n}$, the dominant energy condition is satisfied anywhere.

In this case, we see from (11) that the interior $V^{\prime}(-d)=\frac{2 \sqrt{\alpha}}{n+1} \tan (\sqrt{\alpha} d) \neq$ 0 . Therefore, the solution can only be matched to the Taub's vacuum

$$
\begin{gathered}
d s^{2}=-\left(1+\frac{n+1}{n-1} g_{T}(z+d)\right)^{-2\left(\frac{n-1}{n+1}\right)} d t^{2} \\
+C\left(1+\frac{n+1}{n-1} g_{T}(z+d)\right)^{\frac{4}{n+1}}\left(\left(d x^{1}\right)^{2}+\ldots+\left(d x^{n}\right)^{2}\right)+d z^{2}, \\
-\infty<t<\infty,-\infty<x^{1}<\infty, \ldots,-\infty<x^{n}<\infty,-\frac{n-1}{(n+1) g_{T}}-d<z<-d,
\end{gathered}
$$

which describes a homogeneous gravitational field $+g_{T}$ in the vertical direction and finishes up at an empty singular boundary at $z=-\frac{n-1}{(n+1) g_{T}}-d$.

Since $g_{t t}(-d)=-1$ and $g_{i i}(-d)=C$, by comparing with (21), we see that the continuity of the metric components is assured if we set $C=\cos ^{\frac{4}{n+1}}(\sqrt{\alpha} d)$. And, concerning the derivatives of metric's components, from (11) we get $\mathcal{G}^{\prime}(-d)=-\frac{n-1}{2} V^{\prime}(-d)$, and so their continuity requires

$$
g_{T}=\frac{(n-1)}{n+1} \sqrt{\alpha} \tan (\sqrt{\alpha} d),
$$

which gives the lower external gravitational field $g_{T}$ in terms of matter density $\rho$ and depth $d$.

Now, by eliminating $\kappa$ by means of (28), the solution can be completely parameterized in terms of $d$ and $\rho$, (22) becomes

$$
\begin{array}{r}
\mathcal{G}(z)=\frac{{ }_{2} F_{1}\left(-\frac{1}{2}, \frac{1-n}{2(n+1)} ; \frac{1}{2} ; \sin ^{2}(\sqrt{\alpha} z)\right)}{\cos ^{\frac{n-1}{n+1}}(\sqrt{\alpha} z)}-\frac{\cos ^{\frac{n-1}{n+1}}(\sqrt{\alpha} d)}{\cos ^{\frac{n-1}{n+1}}(\sqrt{\alpha} z)} \frac{\sin (\sqrt{\alpha} z)}{\sin (\sqrt{\alpha} d)} \\
+\frac{{ }_{2} F_{1}\left(-\frac{1}{2}, \frac{1-n}{2(n+1)} ; \frac{1}{2} ; \sin ^{2}(\sqrt{\alpha} d)\right)}{\cos ^{\frac{n-1}{n+1}}(\sqrt{\alpha} z)} \frac{\sin (\sqrt{\alpha} z)}{\sin (\sqrt{\alpha} d)},
\end{array}
$$

the upper gravitational field (27)

$$
g_{R}=\frac{\sqrt{\alpha}}{\sin (\sqrt{\alpha} d)}\left({ }_{2} F_{1}\left(-\frac{1}{2}, \frac{1-n}{2(n+1)} ; \frac{1}{2} ; \sin ^{2}(\sqrt{\alpha} d)\right)-\cos ^{\frac{n-1}{n+1}}(\sqrt{\alpha} d)\right),
$$

and by using (24) and (31) we can write down the pressure.

This solution is remarkably asymmetric, not only because both external gravitational fields are different, as one can see by comparing (30) and (32), but also because the nature of vacua is completely different: the upper one is flat and semi-infinite, whereas the lower one is curved and finishes up down below at an empty repelling boundary where space-time curvature diverges. 
It can be readily seen that, in the upper vacuum, only vertically moving massless particles escape to infinite, whereas non-vertically moving massless ones as well as every massive particle, fall down after reaching a turning point. Consequently, in the lower one, only vertical null geodesics touch the singularity and bounce, whereas non-vertical null ones as well as massive particles bounce before getting to it.

This exact solution clearly shows how the attraction of distant matter can shrink the space-time in such a way that it finishes at an empty singular boundary, as pointed out in [1] and [2]

We can readily see from (30), (31) and (32) that, in the Newtonian limit, i.e., $\sqrt{\rho} d \ll 1$, the mirror symmetry is restored.

For arbitrary $n \geq 2$, we can also match two interior solutions facing each other as discussed in [3,4]. Thus we consider two incompressible fluids joined at $u=\pi / 2$ where the pressure vanishes, the lower one having density $\rho$ and the upper one having density $\rho^{\prime}$. We can readily see that $g_{t t}, g_{i i}$ and $\partial_{z} g_{i i}$ are continuous at the joint. Furthermore, from (25), we see that the continuity of $\partial_{z} g_{t t}$ requires

$$
\sqrt{\rho}\left(\kappa_{\mathrm{crit}}-\kappa\right)=-\sqrt{\rho^{\prime}}\left(\kappa_{\mathrm{crit}}-\kappa^{\prime}\right) .
$$

Therefore, the joining is only possible between an attractive solution and a repulsive one, or between two neutral ones.

Moreover, it is easy to see that we can also insert a slice of arbitrary thickness of the vacuum solution (20) between two such solutions with $\left(\kappa_{\text {crit }}-\right.$ $\kappa)$ of opposite sign, obtaining a relativistic plane "gravitational capacitor".

\section{Concluding remarks}

In order to find out whether empty singular boundaries can arise in higher dimensional Gravity, in this paper, we have constructed an exact and complete (matter matched to vacua) solution of Einstein's equations for arbitrary $n \geq 2$.

This space-time consists of a $(n+2)$-dimensional static and hyperplane symmetric thick slice of matter, with constant and positive energy density $\rho$ and thickness $d$, surrounded by two different vacua.

The solution turns out to be remarkably asymmetric because the nature of both vacua is completely different: the "upper" one is flat and semi-infinite, whereas the "lower" one is curved and finishes up down below at an empty repelling boundary where space-time curvature diverges.

The pressure is positive and bounded, presenting a maximum at an asymmetrical position between the boundaries. We explicitly wrote down the pressure and the external gravitational fields in terms of $\rho$ and $d$. We showed that if $\sqrt{\rho} d$ is small enough, the dominant energy condition is satisfied all over the space-time.

This exact solution clearly show how the attraction of distant matter can shrink the space-time in such a way that it finishes at an empty singular boundary, as pointed out in [1] and [2]. 
Therefore, in spite of the weakening of gravity with the number of dimensions of the space-time [12,13], our solution clearly shows that it is still strong enough to generate empty singular boundaries for arbitrary $n \geq 2$.

\section{A Killing vectors and adapted coordinates}

We want to find coordinates adapted to a hyperplane symmetric distribution of matter. That is, space-time must be invariant under $n$ translations and under rotations in $n(n-1) / 2$ hyperplanes.

More precisely, a $n+2$ dimensional space-time will be said to be $n$-dimensional Euclidean homogenous if it admits the $r=n(n+1) / 2$ parameter group of isometries of the $n$-dimensional Euclidean space $\operatorname{ISO}(n)$.

Since the spacetime admits $n$ mutually conmuting independent motions, we can choose coordinates $x^{i}, z, t$ so that the corresponding Killing vectors are $\xi_{(i)}=\partial_{i}(i=1, \ldots, n)$, and SO

$$
\xi_{(i)}^{k}=\delta_{i}^{k} \quad\left(\begin{array}{c}
k=t, 1, \ldots, n, z \\
i=1, \ldots, n
\end{array}\right) .
$$

The equations of Killing,

$$
\xi^{k} \partial_{k} g_{i j}+g_{k j} \partial_{i} \xi^{k}+g_{i k} \partial_{j} \xi^{k}=0
$$

corresponding to these vectors become

$$
\partial_{k} g_{i j}=0 \quad\left(\begin{array}{c}
i, j=t, 1, \ldots, n, z \\
k=1, \ldots, n
\end{array}\right) .
$$

Hence all the components of the metric tensor depend only on the coordinates $t$ and $z$ and the metric is unaltered by the finite transformation

$$
x^{i} \rightarrow x^{i}+a^{i} \quad(\text { for } i=1, \ldots, n) .
$$

We can take for the remaining $n(n-1) / 2$ motions, the generators $\xi_{(i j)}=x^{i} \partial_{j}-x^{j} \partial_{i}$ $(i<j$ and $i, j=1, \ldots, n)$, so

$$
\partial_{l} \xi_{(i j)}^{k}=\delta_{l}^{i} \delta_{j}^{k}-\delta_{l}^{j} \delta_{i}^{k} \quad\left(\begin{array}{c}
k, l=t, 1, \ldots, n, z \\
i<j \text { and } i, j=1, \ldots, n
\end{array}\right) .
$$

Taking into account (35) and (37) from the equations of Killing (34), we get

$$
g_{j m} \delta_{l}^{i}-g_{i m} \delta_{l}^{j}+g_{l j} \delta_{m}^{i}-g_{l i} \delta_{m}^{j}=0 \quad\left(\begin{array}{c}
m, l=t, 1, \ldots, n, z \\
i<j \text { and } i, j=1, \ldots, n
\end{array}\right) .
$$

From the last equation we readily find

$$
g_{i i}=g_{j j} \quad \text { and } \quad g_{i j}=g_{i t}=g_{i z}=0 \quad(i \neq j \text { and } i, j=1, \ldots, n) .
$$

Furthermore, we can take the bidimensional metric of the $V_{2}$ spaces $x^{i}=$ constant $(i=$ $1, \ldots, n)$ in the conformal flat form. Hence, the most general metric admitting this group of isometries may be written as

$$
d s^{2}=-e^{2 U(z, t)}\left(d t^{2}-d z^{2}\right)+e^{2 V(z, t)}\left(\left(d x^{1}\right)^{2}+\ldots+\left(d x^{n}\right)^{2}\right) .
$$

If, in addition, we impose staticity, $U$ and $V$ must be time independent, and the change of variable $\int e^{U(z)} d z \rightarrow z$ brings the line element to the form (1). 


\section{References}

1. R. E. Gamboa Saraví, Int. J. Mod. Phys. A 23, 1995 (2008).

2. R. E. Gamboa Saraví, Class. Quantum Grav. 25, 045005 (2008).

3. R. E. Gamboa Saraví, Gen. Relativ. Gravit. 41, 1459 (2009).

4. R. E. Gamboa Saraví, Int. J. Mod. Phys. A 24, 5381 (2009).

5. A. H. Taub, Ann. Math. 53, 472 (1951).

6. R. E. Gamboa Saraví, M. Sanmartino, P. Tchamitchian, Class. Quantum Grav. 27, 215016 (2010).

7. A. H. Taub, Phys. Rev. 103, 454 (1956).

8. J. M. Maldacena, Adv.Theor.Math.Phys. 2, 231(1998).

9. O. Aharony, S.S. Gubser, J. Maldacena, H. Ooguri, Y. Oz, Phys. Rept. 323, 183 (2000).

10. R. Maartens, K. Koyama, Living Rev. Relativity, 13, 5 (2010).

11. B. Carter, Phys. Rev. Lett. 26, 331 (1971).

12. F. R. Tangherlini, Nuovo Cimento 27, 636 (1963).

13. J. Ponce de Leon, N. Cruz, Gen. Relativ. Gravit. 32, 1207 (2000).

14. R. C. Myers, M. J. Perry, Ann. Phys. 172, 304 (1986).

15. R. Emparan, H. S. Reall, Living Rev. Relativity, 11, 6 (2008).

16. I. S. Gradshteyn, I. M. Ryzhik, Table of Integrals, Series, and Products (Academic Press Inc., New York, 1963).

17. J. Novotný, J. Horský, Czech. J. Phys. B 24, 718 (1974).

18. C. Liang, J. Math. Phys. 31, 1464 (1990). 